\title{
Synthesis of nano-spherical nickel by templating hibiscus flower petals
}

\author{
Abhijit Kar ${ }^{1, ~ *}$, Ajoy Kumar Ray ${ }^{2}$ \\ ${ }^{1}$ J.B.Centre of Excellence, Jagadis Bose National Science Talent Search, Kolkata, India \\ ${ }^{2}$ Centre for Material Science and Nano Technology Sikkim Manipal Institute of Technology, Sikkim, India
}

Email address:

chatrak130@yahoo.co.in (A. Kar)

To site this article:

Abhijit Kar, Ajoy Kumar Ray. Synthesis of Nano-Spherical Nickel by Templating Hibiscus Flower Petals. American Journal of Nanoscience and Nanotechnology. Vol. 2, No. 2, 2014, pp. 17-20. doi: 10.11648/j.nano.20140202.11

\begin{abstract}
In this study, we have represented synthesis of nano spherical nickel using porous Hibiscus rosa-sinensis petals as bio template. Synthesized Nickel nanoparticle thus characterized by SEM, TEM, XRD and wet chemical methods. Spherical Nickel particles of sized between $10 \mathrm{~nm}$ to $200 \mathrm{~nm}$ was observed.
\end{abstract}

Keywords: Nano Nickel, Catalyst, Bio-Template, Scanning Electron Microscopy, X-Ray Diffraction

\section{Introduction}

Nano-nickel has very high catalytic activity. Unprotected metal nano particle is prone to oxidation and thus conventional methods for the synthesis of metal nanoparticles are not very useful for various applications. As nano metal particle is very prone to oxidation and agglomeration, synthesis of suitably protected metal nanoparticle is found to be difficult and hence different methods are being attempted [1]. It is found that use of capping agents or coating with surfactants may resist the oxidation of nano metal particles. Synthesis of nano nickel particle (un-oxidised and non-agglomerated) is in very high demand as it has diverse applications. By changing the nickel content from 1 to $10 \mathrm{wt} \%$, Lixiong Zhang et al. showed that nano nickel can be used for selective gas permeable membranes [2]. Dye separation performance of the nickel particles embedded ferromagnetic hierarchical porous carbon (FHPC) was investigated by Wang et al. [3]. Nano nickel powder exhibits a strong catalytic effect and can be used in hydrogenation of organic compounds, tail gas processing of vehicle, increase combustion efficiency of solid rocket, reduce sintering temperature etc. it is also useful as magnetically separable catalysts, catalyst supports, and gas adsorbents [4]. Although nano nickel particle has many potential applications, synthesis of these materials were mostly attempted through conventional chemical routes, which involves several steps and use of expensive chemicals including reducing agents, as a result, the widespread application of nano nickel was restricted[5]. Here in this study we have chosen hibiscus flower as soft and porous bio template as well as an in-situ reducing agent while decomposed at higher temperature. This method eventually could produce spherical nickel nano particle along with some mesoporous carbon.

There are several ways of synthesizing mesoporous carbon and nano nickel particles, however, in-situ formation of these materials are not well-established [6, 7, 8]. This paper, aims to report a very simple, cost effective and environmental hazard free green synthesis of spherical nickel nano particle by templating hibiscus flower petals as reducing material.

\section{Experimental}

Hibiscus flower (Hibiscus rosa-sinensis) was collected just before the dawn (when the flowers did not open up completely). Petals were separated out from rest of the flower parts and were very gently washed with distilled water and air dried at room temperature $\left(25^{\circ} \mathrm{C}\right)$ for $\sim 4 \mathrm{hrs}$. to create partial voids (by evaporation of moisture).

A solution of $0.5 \mathrm{M} \mathrm{NiCl}_{2} \cdot 6 \mathrm{H}_{2} \mathrm{O}$, was added into partially air dried petals and allowed to soak for overnight ( $\sim 16 \mathrm{hrs}$.). Ni-salt soaked petals lift off from rest of the $\mathrm{NiCl}_{2} \cdot 6 \mathrm{H}_{2} \mathrm{O}$ solution and were dried under IR lamp $\left(\sim 80^{\circ} \mathrm{C}\right)$ for $4 \mathrm{hrs}$. The $\mathrm{NiCl}_{2}$ incorporated dried petals are placed in a graphite crucible and heated in graphite resistance furnace (ASTRO, Thermal Inc., USA) at $700^{\circ} \mathrm{C}$ under vacuum $\left(\sim 10^{-1} \mathrm{mbar}\right)$ for 30 mins. The rate of heating was maintained $\sim 20^{\circ} \mathrm{C} / \mathrm{min}$. Low vacuum scanning electron microscope (TM1000, 
Hitachi, Japan) was used to see the microstructure of green as well as the dried $\left(\sim 80^{\circ} \mathrm{C}\right)$ petals. The materials heated at $700^{\circ} \mathrm{C}$ were characterization by SEM-EDS, TEM, and $\mathrm{XRD}$. The $\mathrm{wt} \%$ of $\mathrm{Ni}$ in the sample was found out by weight chemical method.

\section{Results and Discussion}

In order to avoid the atmospheric contamination, flowers were plucked in the very early morning and in a fully matured bud, later it was allowed to grow (in few hours) into full flower under clean room condition. The appearance of the pyrolyzed product was black and gets attracted by magnet, thus confirms the presence of $\mathrm{Ni}$ particle. Metallic nickel present in the sintered product could not be separated by magnet even after the sintered product was washed, dried and vibrated ultrasonically. It was interesting to note that size of the cup like pores reduced substantially from top surface to inside of the petals, often there observed partitions within a cup (Fig. 1). As the parts of a petal were porous, the partitioning walls have micro or nano interconnected tunnels that help supply moisture, nutrients and other organic materials from the plant to all parts of the petal. The pores and tunnels beneath the pores have very high capillary action, we have utilised these pores and tunnels for adsorbing $\mathrm{NiCl}_{2} \cdot 6 \mathrm{H}_{2} \mathrm{O}$ solution within it and interconnecting tunnels of the petals.

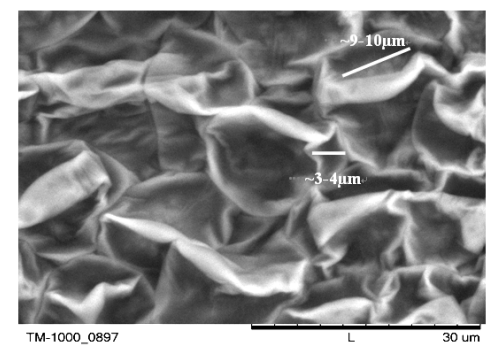

Figure 1. SEM micrograph shows the top surface of fresh hibiscus petal, the cup like pores including the partitions between the pores are separated from each other.

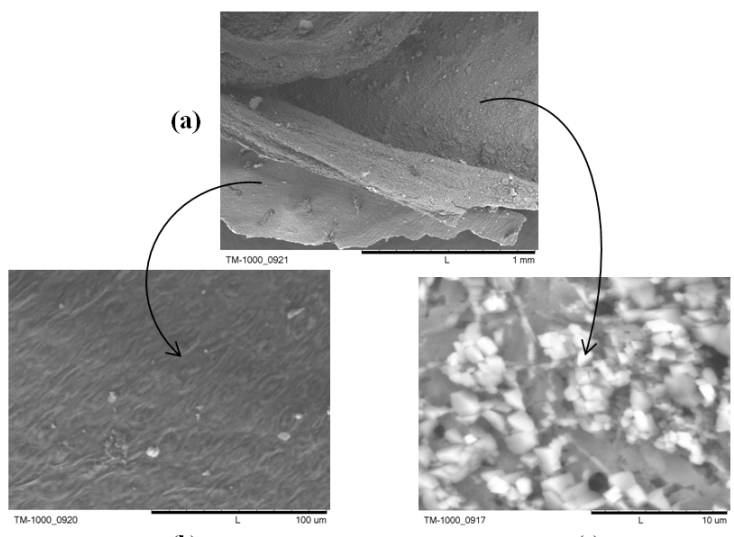

(b)

(c)

Figure 2. SEM micrographs of $\mathrm{NiCl}_{2} \cdot 6 \mathrm{H}_{2} \mathrm{O}$ soaked petals dried at $110^{\circ} \mathrm{C}$ - (a) Micrograph showing both bottom and top surface of a petal, (b) Bottom surface - practically no $\mathrm{NiCl}_{2} \cdot 6 \mathrm{H}_{2} \mathrm{O}$ and (c) Cup shaped pores on the top surface consists of $\mathrm{NiCl}_{2} \cdot 6 \mathrm{H}_{2} \mathrm{O}$ crystals.
Initially when partially dried petals were dipped into the $\mathrm{NiCl}_{2}$ solution, after gentle agitation (with a glass rod) for about 1-2 hrs., petals floats as submerged condition into the $\mathrm{NiCl}_{2}$ solution and the colour changed from red to dirty purple.

This indicates that, $\mathrm{NiCl}_{2}$ diffused into the pores and tunnels of the petals by osmosis phenomena. Microstructure of the bottom surface of the petals, practically does not exhibit porosities, rather it exhibits cellulosic structure (Fig. 2 a-c). Pore sizes of the top surface of the dried petals vary between 1 to $12 \mu \mathrm{m}$ (Fig. 1). If we consider a cup like pore having diameter of $\sim 10 \mu \mathrm{m}$ (as marked in Fig.1), then it could be able to accommodate $6.23 \times 10^{-11} \mathrm{gm} \mathrm{NiCl} 2.6 \mathrm{H}_{2} \mathrm{O}$ equivalent to $1.52 \times 10^{-11} \mathrm{gm} \mathrm{Ni}$, when $0.5 \mathrm{M} \mathrm{NiCl}_{2} .6 \mathrm{H}_{2} \mathrm{O}$ solution was used (density of $\mathrm{Ni}$ is $8.9 \mathrm{~g} / \mathrm{cm}^{3}$ ). Considering the shapes of the cup like pores as a sphere and consequentially the nickel particle formed out of it also spherical, then the final diameter of the spherical nickel formed within the pore should be $\sim 740 \mu \mathrm{m}$. Similarly $1 \mu \mathrm{m}$ diameter cup should yield $0.074 \mu \mathrm{m}$ or $74 \mathrm{~nm}$ diameter Ni particle. Since it was difficult to measure the subsurface pore size we could not calculate lower limit of the Ni particle size. SEM micrograph and EDS analysis (Fig. 3) as well as TEM micrograph and corresponding diffraction patterns (Fig. 4.) showed that there exist good amount of Ni nano particle of size that varies from $10 \mathrm{~nm}$ to $700 \mathrm{~nm}$. Figure 3 exhibits the near spherical $\mathrm{Ni}$ were separated from each other and the inset showed that the Ni balls were entrapped within the pores and tunnels of the pyrolyzed petals. Between the Ni spheres, porous carbon prevents them from agglomeration.

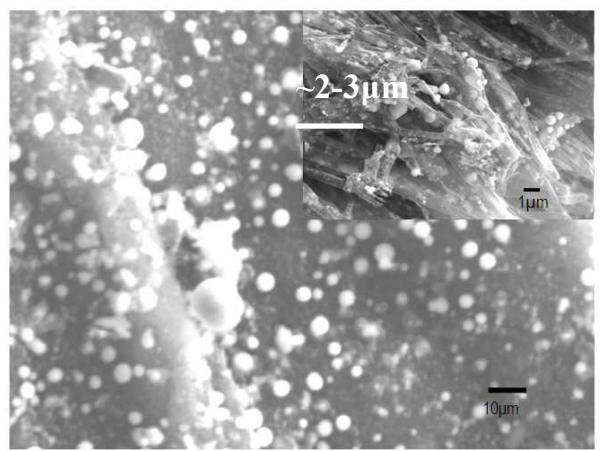

Figure 3. The SEM micrograph and subsequent EDS reveals that plenty of spherical $\mathrm{Ni}$ balls are formed when $\mathrm{NiCl}_{2} \cdot 6 \mathrm{H}_{2} \mathrm{O}$ soaked petals were pyrolysed at $700^{\circ} \mathrm{C}$. In set exhibits the Ni balls are entrapped within the pores and tunnels of the pyrolysed petals.

It was also observed that, even when the heat treatment was carried out at $1000^{\circ} \mathrm{C}, \mathrm{Ni}$ ball did not agglomerate.

During pyrolysis of the $\mathrm{NiCl}_{2} \cdot 6 \mathrm{H}_{2} \mathrm{O}$ soaked petals, various organic constituents of the petals decomposed and released. As of yet, not much information have been reported about the decomposition behavior of hibiscus flower petals. Yong Wang et.al [8] reported that the chemical constitutions of pollen contain several components along with cellulose, and hemicelluloses; 
thermal decomposition of pollen in $\mathrm{N}_{2}$ atmosphere starts $\sim 130^{\circ} \mathrm{C}$, which ends at $\sim 450^{\circ} \mathrm{C}$.

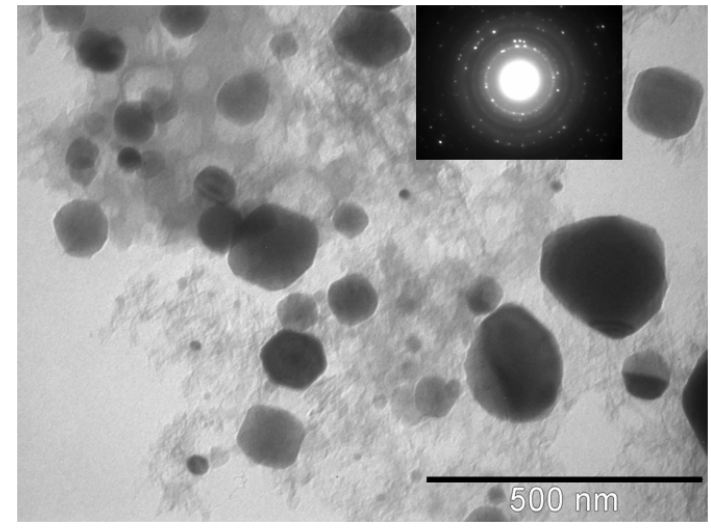

Figure 4. TEM micrograph shows the size of Ni varies from 10 to $200 \mathrm{~nm}$. In the inset corresponding selected area diffraction pattern is shown.

Recently, Yu-Chuan Lin et al. many other researchers reported that pyrolysis of cellulose produces several products including $\mathrm{CO} / \mathrm{C}_{2} \mathrm{H}_{4}(\sim 12.7 \%), \mathrm{CO}_{2}(\sim 19.3 \%)$ and decomposition is dependent on the heating rate [9]. Lanza et al. provided good information about the nature and kinetics of gas evolution when cellulose is pyrolyzed [10, $11,12]$.

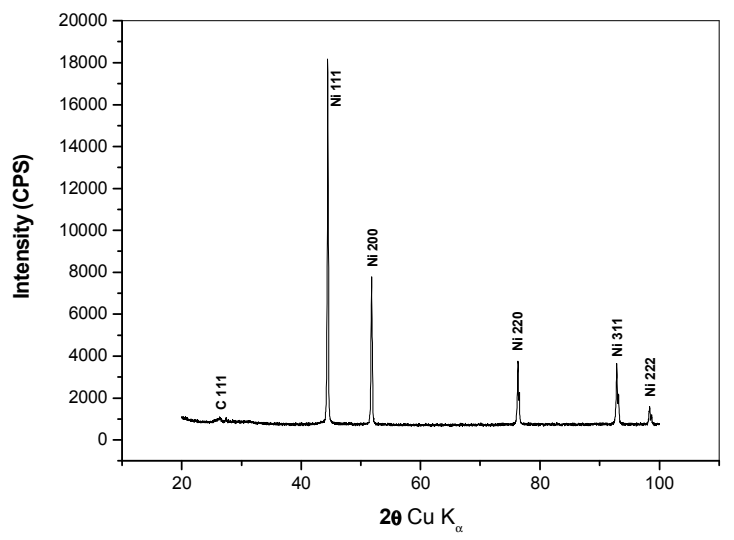

expect $\mathrm{CH}_{3} \mathrm{CHO}$ as one of the reaction product. In order to verify this proposal we did an experiment using a simple glass apparatus, the outlet of the apparatus was dipped into Tollen's reagent. On heating the $\mathrm{NiCl}_{2}$ embedded hibiscus petal, the exhausted gas reduced the Tollen's reagent to form $\mathrm{Ag}$ mirror; on the contrary the petals without soaked in $\mathrm{NiCl}_{2}$ could not reduce the Tollen's reagent. Therefore, it can be concluded that $\mathrm{C}_{2} \mathrm{H}_{4}$, which is the pyrolyzed product of cellulose present in the petals reduced $\mathrm{NiCl}_{2}$ to form $\mathrm{Ni}$, thus follows the reaction below. $\mathrm{NiCl}_{2}+\mathrm{CH}_{2}=\mathrm{CH}_{2}+\mathrm{H}_{2} \mathrm{O} \rightarrow \mathrm{Ni}+\mathrm{CH}_{3} \mathrm{CHO}+$ $2 \mathrm{HClXRD}$ analysis of the pyrolyzed products confirms presence of only metallic Ni and carbon (Fig. 5). Weight chemical analysis confirms that $\sim 37 \mathrm{wt} \%$ of metallic $\mathrm{Ni}$ was present in the products and rest was mesoporous / amorphous carbon. XRD was taken 20 days after the pyrolysis, while material was kept in open atmosphere that means the metallic Ni did not oxidised at least during this period. This indicates that Nano nickel particle produced by this process was coated in situ by carbon so as to prevent its oxidation.

\section{Conclusions}

Porosity of the Hibiscus rosa-sinensis petals could act as a bio template as well as a reducing material for synthesizing the nano nickel particle. Current research demonstrated that the controlled atmosphere pyrolysis of $\mathrm{NiCl}_{2} \cdot 6 \mathrm{H}_{2} \mathrm{O}$ soaked petals could produce nano nickel particles in situ coated with carbon. Size of nano nickel particles varies between $10 \mathrm{~nm}$ to $200 \mathrm{~nm}$.

\section{Acknowledgements}

Authors would like to thank the DST-SERB, Govt. of India for partial financial support and the Director, CSMCRI-Bhavnagar, for allowing us to use their instrumental facilities for conducting some of the analysis of the current research.

Figure 5. XRD analysis of the pyrolysed product shows that the sample contains only Ni and Carbon.

This carbon is considered to be mesoporous carbon and evolved from the hibiscus petals during pyrolysis at $700^{\circ} \mathrm{C}$.According to them maximum amount of $\mathrm{CO}$ evolved at $250^{\circ} \mathrm{C}$ then sequentially $\mathrm{C}_{2} \mathrm{H}_{4}, \mathrm{CH}_{4}, \mathrm{CO}_{2}$ and the least is $\mathrm{H}_{2}$. In the current study thus, either $\mathrm{CO}$ or $\mathrm{C}_{2} \mathrm{H}_{4}$ could have reduced the $\mathrm{NiCl}_{2}$ to $\mathrm{Ni}$ during the pyrolysis of $\mathrm{NiCl}_{2} \cdot 6 \mathrm{H}_{2} \mathrm{O}$ soaked petals. $\mathrm{NiCl}_{2}$ could be reduced by $\mathrm{CO}$ at about $180^{\circ} \mathrm{C}$ to form $\mathrm{Ni}(\mathrm{CO})_{4}$. Subsequently the $\mathrm{Ni}(\mathrm{CO})$ ${ }_{4}$ could produce Ni. However, $\mathrm{Ni}(\mathrm{CO})_{4}$ being highly volatile should go out from the system during the pyrolysis even much before $700^{\circ} \mathrm{C}$. However it was evident from the SEM-EDS analysis (Fig. 3) that good amount of $\mathrm{Ni}$ was present at the surface of the pyrolyzed petals. Hence $\mathrm{CO}$ was not the reducing agent in this case. Whereas, $\mathrm{C}_{2} \mathrm{H}_{4}$ could directly reduce $\mathrm{NiCl}_{2}$ to $\mathrm{Ni}$. In that case one should

\section{References}

[1] E. Veena Gopalan, K. A. Malini, G. Santhoshkumar, T. N. Narayanan, P. A. Joy, I. A. Al-Omari, D. Sakthi Kumar, Yasuhiko Yoshida, M. R. Anantharaman, (2010) TemplateAssisted Synthesis and Characterization of Passivated Nickel Nanoparticles. Nanoscale Res Lett,5:889-897

[2] Lixiong, Z., Xinhua, C., Changfeng Z. and Nanping X., (2006), Preparation and gas separation of nano-sized nickel particle-filled carbon membranes. Journal of Membrane Science, 15: 429-434, Wang, D.W., Li, F., Lu, G. Q., and Cheng, H.M., (2008), Synthesis and dye separation performance of ferromagnetic hierarchical porous carbon. Carbon, 46: 1593-1599.

[4] XiqingWang, Sheng Dai. (2009), A simple method to ordered mesoporous carbons containing nickel nanoparticles. Adsorption, 15:138-144. 
[5] Khanna P.K, Priyesh V. More, Jagdish P. Jawalkar and B.G. Bharate. (2009), Effect of reducing agent on the synthesis of nickel nanoparticles. Materials Letters, 1384-1386

[6] H. Hatori, T. Kobayashi, S. Hishiki, Y. Yamada, S. Matsuno and T. Nishio. (2001), Nano-space structure of carbon from polyimide containing nickel nitrate and their function on catalytic reaction. This article is not included in your organization's subscription. However, you may be able to access this article under your organization's agreement with Elsevier.Synthetic Metals, 125: 183-188

[7] Shaobin Wang and G. Q. (Max) Lu. (1998), Effects of acidic treatments on the pore and surface properties of $\mathrm{Ni}$ catalyst supported on activated carbon. Carbon, 36-3: 283292Yong Wang, Zhimin Liu, Buxing Han, Ying Huang, and Guanying Yang. (2005), Carbon Microspheres with Supported Silver Nanoparticles Prepared from Pollen Grains. Langmuir, 21: 10846-10849
[9] Yu-Chuan Lin, Joungmo Cho, Geoffrey A. Tompsett, Phillip R. Westmoreland, and George W. Huber. (2009), Kinetics and Mechanism of Cellulose Pyrolysis. J. Phys. Chem. C, 113: 20097-20107

[10] Lanza, R., Nogare, D. Dalle, and Canu, P., (2009), Gas Phase Chemistry in Cellulose Fast Pyrolysis. Ind. Eng. Chem. Res, 48:1391-1399

[11] Wagstaff, C., Chanasut, Harren2, U.F. J. M., Laarhoven, L.J., Thomas, B., Rogers, H.J., and Stead, A.D. (2005), Ethylene and flower longevity in Alstroemeria: relationship between tepal senescence, abscission and ethylene biosynthesis. Journal of Experimental Botany, 56:10071016

[12] Michael S. Reid \& Men -Jen Wu. (1992), Ethylene and flower senescence. Plant Growth Regulation, 11:31-43 